\title{
Product innovation with lumpy investment
}

\author{
M. Chahim ${ }^{1}$ • D. Grass ${ }^{2}$ - R. F. Hartl ${ }^{3}$. \\ P. M. Kort $^{4,5}$
}

(C) The Author(s) 2015. This article is published with open access at Springerlink.com

\begin{abstract}
The paper provides a framework that enables us to analyze the important topic of capital accumulation under technological progress. We describe an algorithm to solve Impulse Control problems, based on a (multipoint) boundary value problem approach. Investment takes place in lumps and we determine the optimal timing of technology adoptions as well as the size of the corresponding investments. Our numerical approach led to some guidelines for new technology investments. First, we find that investments are larger and occur in a later stadium when more of the old capital stock needs to be scrapped. Moreover, we obtain that the size of the firm's investments increase when the technology produces more profitable products. We see that the firm
\end{abstract}

\footnotetext{
$\bowtie \quad$ M. Chahim

mohammed.chahim@tno.nl

D. Grass

dieter.grass@tuwien.ac.at

R. F. Hartl

richard.hartl@univie.ac.at

P. M. Kort

kort@tilburguniversity.edu

1 Department of Strategy and Policy, Netherlands Organisation for Applied Scientific Research (TNO), Delft, The Netherlands

2 Department of Operations Research and Systems Theory, Institute of Operations Research and Control Systems (ORCOS), Vienna University of Technology, Vienna, Austria

3 Department of Business Administration, Production and Operations Management, University of Vienna, Vienna, Austria

4 Department of Economics, University of Antwerp, Antwerp, Belgium

5 Department of Econometrics and Operations Research, CentER, Dynamic Optimization in Economics and Operations Research, Tilburg University, Tilburg, The Netherlands
} 
in the beginning of the planning period adopts new technologies faster as time proceeds, but later on the opposite happens. Furthermore, we find that the firm does not invest such that marginal profit is zero, but instead marginal profit is negative.

Keywords (multipoint) Boundary value problem (BVP) - Discrete continuous system · Impulse control maximum principle · Optimal Control · Product innovation · Retrofitting $\cdot$ state-jumps

JEL Classifications C61 $\cdot$ D90 $\cdot 032 \cdot 033$

\section{Introduction}

In today's knowledge economy innovation is of prime importance. Innovation has led to the extraordinary productivity gains in the $1990 \mathrm{~s}$. In current business practice it is felt that the heat is on and that firms must innovate faster just to stand still (The Economist, October 13th 2007, Innovation: Something new under the sun). Therefore, technological progress is a crucial input for firms in taking their investment decisions. Greenwood et al. (1997) argue that technological progress is the main driver of economic growth. They discovered that in the post-war period in the US about $60 \%$ of labor productivity growth was investment specific. Yorokoglu (1998) notes that information technology is a prime example where embodied technological progress led to an improvement of computing technology on the order of 20 times within less than a decade in the 1980s-1990s.

This paper combines technology adoption with capital accumulation, taking into account technological progress. The aim of this paper is to study the decision of when to introduce a new product. To do so we employ the Impulse Control modeling approach that is perfectly suitable to take into account the disruptive changes caused by innovations. This also enables us to determine the length of the time interval that the firm uses a particular technology, when it is time to launch a new product generation, and how these decisions interact with the firm's capital accumulation behavior. In Kort (1989) a dynamic model of the firm is designed in which capital stock jumps upward at discrete points in time at which the firm invests. However, technological progress is not taken into account.

An example where a firm has to decide about investments in new generations of products is the LCD industry. With every new generation the size of the mother glass or substrate increases. As the LCD panels are cut out of the substrate, the substrate on the one hand determines which panel sizes can be produced and on the other hand how efficiently each possible panel size can be produced. We have a process innovation, because a larger glass area provides a more efficient solution of the "cutting problem", and thus lower costs in the production process. A product innovation arises, because the larger area of the substrate makes it possible to produce larger screens. For a firm it is important to determine when it is optimal to introduce a new product. However, since the new product will decrease the demand of the old product, the moment of introduction is crucial. 
Feichtinger et al. (2006) employs a vintage capital goods structure to study the effect of embodied technological progress on the investment behavior of the firm. They show that in the case that a firm has market power a negative anticipation effect occurs, i.e. when technological progress goes faster in the future, it is optimal for the firm to decrease investments in the current generation of capital goods. However, a direct implication of the vintage capital approach is that the firm adopts an infinite amount of different technologies. Of course, in practice a firm can adopt a new technology a limited number of times.

Grass et al. (2012) also combines technology adoption with capital accumulation, while taking into account technological progress. They find that investment jumps upward right at the moment that a new technology is adopted, and that the larger the firm the later the investment in a new technology takes place. Moreover, they find that when a firm has market power, the firm cuts down on investment before a new technology is adopted. Whereas Grass et al. (2012) limits itself to process innovation, we concentrate on studying product innovation. Grass et al. (2012) uses a multi-stage optimal control approach where a firm adopts a new technology in each stage. Unlike Feichtinger et al. (2006), the number of technology adoptions is limited. However, the number of innovations is not determined by the model, but fixed exogenously instead. Unlike Feichtinger et al. (2006) and Grass et al. (2012), in this paper capital accumulation only occurs in lumps. Moreover, these lumps are determined by the model, i.e. the lumpy investments are endogenous. In Saglam (2011) a multi-stage optimal control model is studied where the number of technology adoptions is endogenous. However, unlike our paper, the model does not incorporate any (fixed) cost associated with the adoption and the considered firm has no market power. In Boucekkine et al. (2004) a two-stage optimal control model is considered, where only one adoption occurs, without adoption (fixed) cost. Both Boucekkine et al. (2004) and Saglam (2011) incorporate learning, were the firm raises productivity of a given technology over time due to learning and revenue is linear in the capital stock.

Our paper is mostly comparable to Grass et al. (2012). However, unlike Grass et al. (2012), we can endogenously determine the number of technology adoptions over the planning period and we do incorporate a fixed cost associated with a technology adoption. However, the most significant difference with Grass et al. (2012) is that in the present model investment takes place in lumps. The resulting upward jump in the capital stock approaches reality more than a continuous development of capital stock over time, the latter being the result of the analysis in Grass et al. (2012).

The method used to study firm behavior in this paper is Impulse Control. Impulse Control theory is a variant of optimal control theory where discontinuities (i.e. jumps) in the state variable are allowed. In Impulse Control the moments of these jumps as well as the sizes of the jumps are decision variables. Blaquière (1977a, b, 1979, 1985) extends the standard theory on optimal control by deriving a Maximum Principle, the so-called Impulse Control Maximum Principle, that gives necessary and sufficient optimality conditions for solving such problems. Blaquière's Impulse Control analysis is based on the present value Hamiltonian form. In this paper we apply the Impulse Control theorem in the current value Hamiltonian framework as derived in Chahim et al. (2012). 
One of the striking results is that the firm does not invest such that the marginal profit is zero, but instead marginal profit is negative. Furthermore, we obtain that the firm in the beginning of the planning period adopts new technologies faster as time proceeds, but after some moment in time later technologies are used for a longer time period. This behavior is different from Grass et al. (2012), who finds that the firm adopts new technologies faster as time proceeds for the whole planning period, but this also differs from the results found in Saglam (2011), who finds that later technologies are used during a longer time period. Our results are somehow a combination of both.

This paper is organized as follows. In Sect. 2 we briefly introduce Impulse Control. Section 3 gives the general setting and builds up the product innovation Impulse Control model. Section 4 derives the necessary optimality conditions for the product innovation problem, whereas Sect. 5 gives a brief description of the algorithms present in the literature dealing with the Impulse Control Maximum Principle and describes an algorithm to solve Impulse Control problems, based on a (multipoint) boundary value problem (BVP) approach. In Sect. 6 we study the investment behavior of a product innovating firm, and in Sect. 7 we extend this analysis by adding decreasing demand, i.e. demand decreases over time due to competitors producing better products because of technological progress. Finally, in Sect. 8 we conclude and give some recommendations for future research.

\section{A brief introduction to impulse control}

In this section we introduce a general impulse control model and provide necessary optimality conditions.

Let us denote $x$ as the state variable, $u$ as an ordinary control variable and $v^{i}$ as the impulse control variable, where $x$ and $u$ are piecewise continuous functions of time. ${ }^{1}$ We denote $r$ as the discount rate leading to the discount factor $e^{-r t}$ at time $t$. The terminal time or horizon date of the system or process is denoted by $T>0$, and $x\left(T^{+}\right)$stands for the state value immediately after a possible jump at time $T$. The profit of the system between jumps is given by $F(x, u, t)$, whereas $G(x, v, t)$ is the profit function associated with a jump, and $S\left(x\left(T^{+}\right)\right)$is the salvage value, i.e. the total costs or profit associated with the system after time $T$. Finally, $f(x, u, t)$ describes the continuous change of the state variable over time between the jump points and $g(x, v, t)$ is a function that represents the instantaneous (finite) change of the state variable when there is an impulse or jump.

The above results in the following optimal control problem

$$
\begin{gathered}
\max _{u(\cdot), N, \tau_{i}, v^{i}}\left\{\int_{0}^{T} \mathrm{e}^{-r t} F(x(t), u(t), t) \mathrm{d} t+\sum_{i=1}^{N} \mathrm{e}^{-r \tau_{i}} G\left(x\left(\tau_{i}^{-}\right), v^{i}, \tau_{i}\right)+\mathrm{e}^{-r T} S\left(x\left(T^{+}\right)\right)\right\}, \\
\text {s.t. } \dot{x}(t)=f(x(t), u(t), t), \\
\text { for } t \in[0, T] \backslash\left\{\tau_{1}, \ldots, \tau_{N}\right\},
\end{gathered}
$$

\footnotetext{
1 Note that the necessary optimality conditions presented in Theorem 1 also hold for measurable controls. Applications typically have piecewise continuous functions.
} 
$x\left(\tau_{i}^{+}\right)-x\left(\tau_{i}^{-}\right)=g\left(x\left(\tau_{i}^{-}\right), v^{i}, \tau_{i}\right)$, for $i \in\{1, \ldots, N\}$,

$x\left(0^{-}\right)=x_{0}, \quad u(t) \in \mathcal{U}, \quad v^{i} \in \mathcal{V}, \quad i \in\{1, \ldots, N\}$.

For $N \in \mathbb{N}$ we assume the jump times to be sorted as

$$
\begin{aligned}
& \tau_{i} \in[0, T] \text { with } 0 \leq \tau_{1}<\cdots<\tau_{N} \leq T, \\
& x\left(\tau_{i}^{+}\right)=\lim _{t \downarrow \tau_{i}} x(t) \text { and } x\left(\tau_{i}^{-}\right)=\lim _{t \uparrow \tau_{i}} x(t), \text { for } i=1, \ldots, N,
\end{aligned}
$$

and

$$
x_{0} \in \mathbb{R}^{n} \text {. }
$$

We assume that the domains $\mathcal{U} \subset \mathbb{R}^{m}$ and $\mathcal{V} \subset \mathbb{R}^{l}$ are bounded convex sets. Further we impose that $F, f, g$ and $G$ are continuously differentiable in $x$ on $\mathbb{R}^{n}$ and $v^{i}$ on $\mathcal{V}$, $S(x)$ is continuously differentiable in $x$ on $\mathbb{R}^{n}$, and that $g$ and $G$ are continuous in $\tau$. Finally, when there is no jump, i.e. $v=0$, we assume that

$$
g(x, 0, t)=0,
$$

for all $x$ and $t$.

\subsection{Necessary optimality conditions}

We apply the impulse control maximum principle in current value formulation in normal form derived in Chahim et al. (2012) to (1a)-(1e). ${ }^{2}$ The resulting necessary optimality conditions are presented in Theorem 1 .

Before we state Theorem 1 , let us define the Hamiltonian $\mathcal{H}$ and the Impulse Hamiltonian $\mathcal{I H}$ by

$$
\begin{aligned}
& \mathcal{H}(x, u, \lambda, t):=F(x, u, t)+\lambda f(x, u, t), \\
& \mathcal{I H}(x, v, \lambda, t):=G(x, v, t)+\lambda g(x, v, t),
\end{aligned}
$$

and define the following abbreviations

$$
\begin{aligned}
& \mathcal{H}[s]:=\mathcal{H}(x(s), u(s), \lambda(s), s), \\
& \mathcal{I} \mathcal{H}[s, v]:=\mathcal{I} \mathcal{H}\left(x\left(s^{-}\right), v, \lambda\left(s^{+}\right), s\right), \\
& G[s, v]:=G\left(x\left(s^{-}\right), v, s\right), \\
& g[s, v]:=g\left(x\left(s^{-}\right), v, s\right) .
\end{aligned}
$$

\footnotetext{
2 Other references deriving the necessary optimality conditions for the Impulse Control problems are Blaquière (1977a,b, 1979, 1985), Seierstad (1981) and Seierstad and Sydsæter (1987).
} 
Theorem 1 (Impulse control maximum principle) Let for $N \in \mathbb{N}$ with $N>0$ $\left(x^{*}(\cdot), u^{*}(\cdot), N, \tau_{1}^{*}, \ldots, \tau_{N}^{*}, v^{1 *}, \ldots, v^{N *}\right)$ be an optimal solution of $(1)$. Then there exists a (piecewise absolutely continuous) adjoint variable $\lambda(\cdot)$ such that the following conditions hold:

$$
\begin{aligned}
& u^{*}(t) \in \underset{u}{\operatorname{argmax}} \mathcal{H}\left(x^{*}(t), u, \lambda(t), t\right), \quad t \in[0, T], \\
& \dot{\lambda}(t)=r \lambda(t)-\frac{\partial}{\partial x} \mathcal{H}\left(x^{*}(t), u^{*}(t), \lambda(t), t\right), \quad t \in[0, T] \backslash\left\{\tau_{1}^{*}, \ldots, \tau_{N}^{*}\right\} .
\end{aligned}
$$

For every $t=\tau_{i}^{*},(i=1, \ldots N)$, we have

$$
\begin{aligned}
& \frac{\partial}{\partial v} \mathcal{I} \mathcal{H}\left(x^{*}\left(\tau_{i}^{*-}\right), v^{i *}, \lambda\left(\tau_{i}^{*+}\right), \tau_{i}^{*}\right)\left(v-v^{i *}\right) \leq 0, \quad v \in \mathcal{V}, \\
& \lambda\left(\tau_{i}^{*+}\right)-\lambda\left(\tau_{i}^{*-}\right)=-\frac{\partial}{\partial x} \mathcal{I} \mathcal{H}\left(x^{*}\left(\tau_{i}^{*-}\right), v^{i *}, \lambda\left(\tau_{i}^{*+}\right), \tau_{i}^{*}\right), \\
& \mathcal{H}\left[\tau_{i}^{*+}\right]-\mathcal{H}\left[\tau_{i}^{*-}\right]+r G\left[\tau_{i}^{*}, v^{i *}\right]-\frac{\partial}{\partial \tau} \mathcal{I} \mathcal{H}\left[\tau_{i}^{*}, v^{i *}\right] \begin{cases}\geq 0 & \tau_{i}^{*}=0 \\
=0 & \tau_{i}^{*} \in(0, T) \\
\leq 0 & \tau_{i}^{*}=T .\end{cases}
\end{aligned}
$$

For $t \in[0, T] \backslash\left\{\tau_{1}^{*}, \ldots, \tau_{N}^{*}\right\}$ it holds that

$$
\frac{\partial}{\partial v} \mathcal{I} \mathcal{H}\left(x^{*}(t), 0, \lambda(t), t\right) v \leq 0, \quad v \in \mathcal{V}
$$

The transversality condition is

$$
\lambda\left(T^{+}\right)=\frac{\partial}{\partial x} S\left(x^{*}\left(T^{+}\right)\right) .
$$

Proof See Blaquière (1977a, 1985).

\section{The product innovation model}

Consider a firm that invests in lumps over time. Each time it invests it installs a production plant suitable to produce the new product. Due to product innovation the quality of the products, and thus also demand, increases over time. This implies that the later an investment takes place, the better products can be made due to these investments.

This is formalized as follows. A plant being installed at time $\tau$ will make products for which the price is given by the following inverse demand function:

$$
p(t)=\theta(\tau)-q(t)
$$


where $q(t)$ is the output at time $t$ and $\theta(\tau)=1+b \tau$ is the state of technology that the firm adopts at time $\tau .{ }^{3}$ We further assume that technology within the firm does not change between two technology adoptions, i.e. $\dot{\theta}=0$ for all $t \neq \tau$. At the moment the firm adopts a technology, the firm's technology change is given by

$$
\theta\left(\tau_{i}^{+}\right)-\theta\left(\tau_{i}^{-}\right)=1+b \tau_{i}-\theta\left(\tau_{i}^{-}\right)=b\left(\tau_{i}-\tau_{i}^{-}\right)=b\left(\tau_{i}-\tau_{i-1}\right) .
$$

Hence, as in Feichtinger et al. (2006) and Grass et al. (2012), we impose that technological progress increases linearly over time, where $b$ is a positive constant. In Feichtinger et al. (2006) it is argued that this holds when we consider the case that technological progress is based on Moore's law, implying that technology develops in an exponential way over time. On the other hand, a Philips manager ${ }^{4}$ argued that utility is a logarithmic function of technology. In total this results in a linear increase of technological progress. In Saglam (2011) technology increases exponentially over time and in Boucekkine et al. (2004) there are only two different technologies available. We assume a simple production function in the sense that one capital good produces one unit of output. Denoting the stock of capital goods by $K(t)$, this gives

$$
K(t)=q(t) .
$$

We impose that only the capital stock of the new plant is able to produce the new products, i.e. each plant has its own capital stock that produces the products with a quality associated with the timing of the investment in this plant. In this setting we can also model a situation where just $100 \gamma \%$, where $\gamma \in[0,1]$, of the capital stock is scrapped, while the remaining machines or tools can be reused for the new product. Hence, full scrapping corresponds to the case where $\gamma=1$. This implies that old products, and thus also old capital goods, become worthless after the new plant is installed, implying that the old capital goods can be scrapped.

Denoting investment by $I(t)$, at the moment the firm invests (adopts a new technology) capital stock changes by

$$
K\left(\tau^{+}\right)-K\left(\tau^{-}\right)=I(\tau)-\gamma K\left(\tau^{-}\right) .
$$

At time zero the capital stock is equal to zero, i.e.

$$
K(0)=0
$$

For each plant it holds that capital stock depreciates with rate $\delta$, i.e.

$$
\dot{K}=-\delta K \text {. }
$$

Investing in a plant implies that the firm has to pay a fixed cost, i.e. part of the cost is independent of the plant size, and a variable cost that more than proportionally

\footnotetext{
3 We assume that technology is continuously changing, i.e. $\theta(t)=1+b t$. However, the technology within the firm is the technology that the firm adopts at time $\tau$.

4 Theo Claassen in the Dutch magazine Elsevier (January 24, 1998).
} 
increases with the size of the plant. In particular, we assume that the investment cost is given by

$$
C(I)=\left\{\begin{array}{cl}
C+\alpha I+\beta I^{2} & \text { for } I>0 \\
0 & \text { for } I=0
\end{array}\right.
$$

This type of investment cost function, but without the fixed cost, is common in the literature (e.g., among others, see Grass et al. (2012) and Sethi and Thompson (2006, pp. 83-88)), in which besides the fixed cost, the linear term consist of acquisition cost, where the unit price is equal to $\alpha$ and the quadratic term represents the adjustment cost. Instead of a quadratic term, any other convexly increasing function could have been imposed.

Total discounted revenue is given by

$$
\int_{0}^{T} e^{-r t}[\theta(t)-K(t)] K(t) d t,
$$

where instantaneous revenue is determined by output price times output. Concerning the objective (6) please note that $\theta(t)$ is constant in the time interval between two jumps in such a way that it equals its value obtained at the jump, when the interval starts. This means that on the time interval $\left(\tau_{i}, \tau_{i+1}\right)$ it holds that $\theta(t)=\theta\left(\tau_{i}\right)$. Since we have a finite time planning period, a salvage value has to be defined. This salvage value is equal to the value of the firm at the time horizon $T$. We assume that this value is given by

$$
+e^{-r T} \frac{\left[\theta\left(\tau_{N}\right)-K\left(T^{+}\right)\right] K\left(T^{+}\right)}{r+\delta} .
$$

The salvage value (7) is a lower bound of the discounted revenue stream of the firm after the planning period.

Total discounted investment cost is given by the sum of the cost of adopting a new technology, discounted at the time the adoption takes place. This results in

$$
-\sum_{i=1}^{N} e^{-r \tau_{i}} C\left(I\left(\tau_{i}\right)\right) .
$$

The above gives rise to the following impulse control model:

$$
\begin{gathered}
\max _{I(\cdot), \tau_{i}, N}\left\{\int_{0}^{T} e^{-r t}[\theta(t)-K(t)] K(t) d t-\sum_{i=1}^{N} e^{-r \tau_{i}} C\left(I\left(\tau_{i}\right)\right)\right. \\
\left.+e^{-r T} \frac{\left[\theta\left(\tau_{N}\right)-K\left(T^{+}\right)\right] K\left(T^{+}\right)}{r+\delta}\right\},
\end{gathered}
$$


subject to

$$
\begin{aligned}
\dot{K}(t) & =-\delta K(t) \text { for all } t \neq \tau_{1}, \ldots, \tau_{N}, \\
\dot{\theta} & =0 \text { for all } t \neq \tau_{1}, \ldots, \tau_{N}, \\
K\left(\tau_{i}^{+}\right)-K\left(\tau_{i}^{-}\right) & =I\left(\tau_{i}\right)-\gamma K\left(\tau_{i}^{-}\right) \text {for all } i=1, \ldots, N \\
\theta\left(\tau_{i}^{+}\right)-\theta\left(\tau_{i}^{-}\right) & =1+b \tau_{i}-\theta\left(\tau_{i}^{-}\right) \text {for all } i=1, \ldots, N \\
K(0) & =0 \\
\theta(0) & =1 .
\end{aligned}
$$

This is an Impulse Control problem as described in Blaquière (1977a, b, 1979, 1985). Note that this innovation model only contains an impulse control variable and no ordinary control variable. This approach differs from the multi-stage approach used in Grass et al. (2012), because here investment takes place in lumps and every investment goes along with a fixed cost. As in Grass et al. (2012) we can model all situations between the extreme cases where after every new investment the old capital goods are scrapped $(\gamma=1)$ and where all the capital can be kept $(\gamma=0)$ to produce the new product. Another benefit of the above model compared to Grass et al. (2012) is that the number of technology adoptions over the planning period is endogenously determined.

\section{Necessary optimality conditions for the product innovation problem}

We apply the impulse control maximum principle in current value formulation derived in Chahim et al. (2012). Other good references deriving the necessary optimality conditions for the Impulse Control problems are Blaquière (1977a, b, 1979, 1985), Seierstad (1981) and Seierstad and Sydsæter (1987). We define the Hamiltonian $\mathcal{H}$ and the Impulse Hamiltonian $\mathcal{I H}$ for the product innovation problem as

$$
\begin{aligned}
\mathcal{H}[t]= & {[\theta(t)-K(t)] K(t)-\lambda_{1}(t) \delta K(t), } \\
\mathcal{I H}\left[\tau_{i}\right]= & -C-\alpha I\left(\tau_{i}\right)-\beta I\left(\tau_{i}\right)^{2}+\lambda_{1}\left(I\left(\tau_{i}\right)-\gamma K\left(\tau_{i}^{-}\right)\right) \\
& +\lambda_{2}\left(1+b \tau_{i}-\theta\left(\tau_{i}^{-}\right)\right),
\end{aligned}
$$

and obtain the adjoint equations

$$
\begin{aligned}
& \dot{\lambda}_{1}(t)=(r+\delta) \lambda_{1}(t)-\theta(t)+2 K(t), \\
& \dot{\lambda}_{2}(t)=r \lambda_{2}(t)-K(t) .
\end{aligned}
$$

The jump conditions are

$$
\begin{aligned}
-\alpha-2 \beta I\left(\tau_{i}\right)+\lambda_{1}\left(\tau_{i}^{+}\right) & =0 \\
\lambda_{1}\left(\tau_{i}^{+}\right)-\lambda_{1}\left(\tau_{i}^{-}\right) & =\gamma \lambda_{1}\left(\tau_{i}^{+}\right), \\
\lambda_{2}\left(\tau_{i}^{+}\right)-\lambda_{2}\left(\tau_{i}^{-}\right) & =\lambda_{2}\left(\tau_{i}^{+}\right),
\end{aligned}
$$


from which we conclude that

$$
\lambda_{1}\left(\tau_{i}^{-}\right)=(1-\gamma) \lambda_{1}\left(\tau_{i}^{+}\right)
$$

which equals zero for $\gamma=1$, and

$$
\lambda_{2}\left(\tau_{i}^{-}\right)=0
$$

The condition for determining the optimal switching time $\tau_{i}$ is

$$
\begin{aligned}
& \mathcal{H}\left[\tau_{i}^{+}\right]-\mathcal{H}\left[\tau_{i}^{-}\right]-\frac{\partial G\left(x\left(\tau_{i}^{-}\right), v_{i}, \lambda\left(\tau_{i}^{+}\right), \tau_{i}\right)}{\partial \tau}+r G\left(x\left(\tau_{i}^{-}\right), v_{i}, \lambda\left(\tau_{i}^{+}\right), \tau_{i}\right) \\
& -\lambda\left(\tau_{i}^{+}\right) \frac{\partial g\left[\tau_{i}^{-}\right]}{\partial \tau} \begin{cases}\geq 0 & \text { for } \tau_{i}^{*}=0 \\
=0 & \text { for } \tau_{i}^{*} \in(0, T) \\
\leq 0 & \text { for } \tau_{i}^{*}=T .\end{cases}
\end{aligned}
$$

Using the above specification, we get

$$
\begin{aligned}
& {\left[\theta\left(\tau_{i}^{+}\right)-K\left(\tau_{i}^{+}\right)\right] K\left(\tau_{i}^{+}\right)-\left[\theta\left(\tau_{i}^{-}\right)-K\left(\tau_{i}^{-}\right)\right] K\left(\tau_{i}^{-}\right)} \\
& -\lambda_{1}\left(\tau_{i}^{+}\right) \delta K\left(\tau_{i}^{+}\right)+\lambda_{1}\left(\tau_{i}^{-}\right) \delta K\left(\tau_{i}^{-}\right)-r C-r \alpha I\left(\tau_{i}\right)-r \beta I\left(\tau_{i}\right)^{2}-b \lambda_{2}\left(\tau_{i}^{+}\right) \\
& \begin{cases}\geq 0 & \text { for } \tau_{i}^{*}=0 \\
=0 & \text { for } \tau_{i}^{*} \in(0, T) \\
\leq 0 & \text { for } \tau_{i}^{*}=T .\end{cases}
\end{aligned}
$$

The transversality conditions are

$$
\begin{aligned}
& \lambda_{1}\left(T^{+}\right)=\frac{\theta\left(\tau_{N}\right)-2 K\left(T^{+}\right)}{r+\delta} \\
& \lambda_{2}\left(T^{+}\right)=\frac{K\left(T^{+}\right)}{r+\delta .}
\end{aligned}
$$

At the non-jump points $t \neq \tau_{1}, \ldots, \tau_{N}$, it holds that $\lim _{I \rightarrow 0} \frac{\partial \mathcal{I H}}{\partial I}=\infty$ due to the fixed cost. Hence, the conditions for applying the Impulse Control Maximum Principle are met, see Section 2.3 of Chahim et al. (2012).

\section{Algorithm}

In the literature three different algorithms are derived based on the Impulse Control Principle (Blaquière (1977a, b, 1979, 1985) and Chahim et al. (2012)). Luhmer (1986) derived a forward algorithm (starts at time 0) and Kort (1989, pp. 62-70) derived a backward algorithm (starts at final time horizon $T$ ). Luhmer (1986) starts at $t=0$ and uses the costate variable, as input to initialize his algorithm. Kort (1989) implements a backward algorithm that starts at the time horizon, i.e. $t=T$, and initializes the algorithm using the values of the state variables. Finally, Grass and Chahim (2012) 
designs an algorithm that is a combination of continuation techniques and a multi-point Boundary Value Problem (BVP) to solve Impulse Control problems.

The problem described by (9)-(15) has two state variables, the stock of capital $(K(t))$ and technology $(\theta(t))$. The question is which algorithm is most suitable for this model. Applying the forward algorithm to problem (9)-(15) has a drawback. Namely, we have to guess the initial values for the two costate variables, $\lambda_{1}$ and $\lambda_{2}$. A wrong guess of the costate variables at the initial time results in a solution that does not satisfy the transversality conditions (24) and (25), which implies that the necessary optimality conditions are not satisfied. For the backward algorithm we start with choosing values for the state variables at time $T$. The resulting solution always satisfies the necessary optimality conditions, but here the problem is that the algorithm has to end up at the right $K(0)$ and $\theta(0)$. In other words, with the backward algorithm one can apply the right necessary conditions to the wrong problem. An example where the backward algorithm is applied successfully is Chahim et al. (2013). Moreover, in Chahim et al. (2013) clear upper and lower bounds have been derived for the state variable.

In addition, the backward algorithm has another drawback. When we apply it to the problem described by (9)-(15), starting at the time horizon and going back in time requires knowledge of the technology before the investment. In particular, we obtain from equation (23) that we need to know $\theta\left(\tau_{N}^{+}\right)=1+b \tau_{N}$ and $\theta\left(\tau_{N}^{-}\right)=\theta\left(\tau_{N-1}\right)=$ $1+b \tau_{N-1}$. Hence, solving this equation for $\tau_{N}$ requires that we know $\tau_{N-1}$. And with the backward algorithm, this predecessor is not known. We conclude that the backward algorithm is not suitable to solve our model.

\section{1 (multipoint) Boundary value approach}

In this section we describe a (multipoint) boundary value problem (BVP), that is useful to solve Impulse Control problems. The idea behind the boundary value approach is that at the time interval between two jumps the system of differential equations (canonical system) combined with the boundary conditions (initial and final conditions) is solved. After each found jump the (multipoint) BVP is updated to find the next jump.

To simplify the presentation and to concentrate on the main concepts of the numerical algorithm, we make the following assumptions.

Assumption 1 For every time horizon $T \geq 0$ there exists a unique optimal solution of (1), with a finite number of jumps (which in general depends on $T$ ).

Assumption 2 Let for $T>0$ the jump times be $\left(\tau_{i}\right)_{i=1}^{N}$ with $0<\tau_{1}<\ldots<\tau_{N}<T$, and $\bar{x}(T):=\left(x\left(\tau_{1}^{-}\right), x\left(\tau_{1}^{+}\right), v_{1}, \ldots, x\left(\tau_{N}^{-}\right), x\left(\tau_{N}^{+}\right), v_{N}\right)$ be the vector of left and right limits of the states together with the optimal impulse control values for the given time horizon $T$. Then in a neighborhood of $T$ the solution vector $\bar{x}(T)$ is continuous.

For the algorithm $T$ is a continuation variable. During the continuation process $T$ is increased and the conditions for possible jumps are monitored. 
Assumption 3 Condition (3c) implies

$$
\frac{\partial}{\partial v} \mathcal{I} \mathcal{H}\left(x^{*}\left(\tau_{i}^{*-}\right), v^{i *}, \lambda\left(\tau_{i}^{*+}\right), \tau_{i}^{*}\right)=0,
$$

and with $\frac{\partial^{2}}{\partial v^{2}} \mathcal{I} \mathcal{H}\left(x^{*}\left(\tau_{i}^{*-}\right), v^{i *}, \lambda\left(\tau_{i}^{*+}\right), \tau_{i}^{*}\right)<0$ this yields

$$
v^{i *}=v\left(x^{*}\left(\tau_{i}^{*-}\right), \lambda\left(\tau_{i}^{*+}\right), \tau_{i}^{*}\right) .
$$

In general condition (3c) does not imply that the optimal impulse control value can be found as the arg max of the Impulse Hamiltonian. However, for the model in this paper it holds, since the $\mathcal{I H}$ is concave for positive $I$.

To formulate the (multipoint) BVP we introduce the following notation for the canonical system dynamics:

$$
\begin{aligned}
& \dot{x}(t)=h_{1}(x(t), \lambda(t), t), \\
& \dot{\lambda}(t)=h_{2}(x(t), \lambda(t), t) .
\end{aligned}
$$

For the conditions at a jumping time $\tau$ we define:

$$
\begin{aligned}
& j^{x}\left(x\left(\tau^{+}\right), x\left(\tau^{-}\right), \lambda\left(\tau^{+}\right), \tau\right):=x\left(\tau^{+}\right)-x\left(\tau^{-}\right)-g\left[\tau, x\left(\tau^{+}\right)-x\left(\tau^{-}\right)\right], \\
& j^{\lambda}\left(x\left(\tau^{-}\right), \lambda\left(\tau^{+}\right), \lambda\left(\tau^{-}\right), \tau\right):=\lambda\left(\tau^{+}\right)-\lambda\left(\tau^{-}\right)+\frac{\partial}{\partial x} \mathcal{I} \mathcal{H}\left[\tau, x\left(\tau^{+}\right)-x\left(\tau^{-}\right)\right], \\
& j^{\tau}\left(x\left(\tau^{-}\right), x\left(\tau^{+}\right), \lambda\left(\tau^{+}\right), \lambda\left(\tau^{-}\right), \tau\right):=\mathcal{H}\left[\tau^{+}\right]-\mathcal{H}\left[\tau^{-}\right]+r G[\tau, v] \\
& \quad-\frac{\partial}{\partial \tau} \mathcal{I} \mathcal{H}\left[\tau, x\left(\tau^{+}\right)-x\left(\tau^{-}\right)\right] .
\end{aligned}
$$

Now let $\left(x^{*}(\cdot), u^{*}(\cdot), N, \tau_{1}^{*}, \ldots, \tau_{N}^{*}, v^{1 *}, \ldots, v^{N *}\right)$ be the optimal solution of (1) with $0<\tau_{1}^{*}<\ldots<\tau_{N}^{*}<T$. Then the necessary conditions yield the following (multipoint) BVP:

$$
\begin{aligned}
& \dot{x}_{i}(t)=h_{1}\left(x_{i}(t), \lambda_{i}(t), t\right), \quad t \in\left[\tau_{i-1}, \tau_{i}\right], i=1, \ldots, N+1, \\
& \dot{\lambda}_{i}(t)=h_{2}\left(x_{i}(t), \lambda_{i}(t), t\right), \quad t \in\left[\tau_{i-1}, \tau_{i}\right], i=1, \ldots, N+1, \\
& j^{x}\left(x_{i}\left(\tau_{i}^{+}\right), x_{i}\left(\tau_{i}^{-}\right), \lambda_{i}\left(\tau_{i}^{+}\right), \tau_{i}\right)=0, \quad i=1, \ldots, N, \\
& j^{\lambda}\left(x_{i}\left(\tau_{i}^{-}\right), \lambda_{i}\left(\tau_{i}^{+}\right), \lambda_{i}\left(\tau_{i}^{-}\right), \tau_{i}\right)=0, \quad i=1, \ldots, N, \\
& j^{\tau_{i}}\left(x_{i}\left(\tau_{i}^{-}\right), x_{i}\left(\tau_{i}^{+}\right), \lambda_{i}\left(\tau_{i}^{+}\right), \lambda_{i}\left(\tau_{i}^{-}\right), \tau_{i}\right)=0, \quad i=1, \ldots, N, \\
& \mathcal{S}\left(x_{N+1}(T), \lambda_{N+1}(T)\right)=0, \\
& x_{1}(0)-x_{0}=0,
\end{aligned}
$$

where (29f) denotes the transversality condition (3g), $\tau_{0}=0$ and $\tau_{N+1}=T$. 
After defining $t(s):=\tau_{i}-(i-s) \Delta \tau_{i}$, with $\Delta \tau_{i}:=\tau_{i}-\tau_{i-1}$, we rewrite (29) into

$$
\begin{aligned}
& \dot{x}_{i}(s)=\Delta \tau_{i} h_{1}\left(x_{i}(s), \lambda_{i}(s), t(s)\right), \quad s \in[i-1, i], i=1, \ldots, N+1, \\
& \dot{\lambda}_{i}(s)=\Delta \tau_{i} h_{2}\left(x_{i}(s), \lambda_{i}(s), t(s)\right), \quad s \in[i-1, i], i=1, \ldots, N+1, \\
& j^{x}\left(x_{i}\left(i^{+}\right), x_{i}\left(i^{-}\right), \lambda_{i}\left(i^{+}\right), \tau_{i}\right)=0, \quad i=1, \ldots, N, \\
& j^{\lambda}\left(x_{i}\left(i^{-}\right), \lambda_{i}\left(i^{+}\right), \lambda_{i}\left(i^{-}\right), \tau_{i}\right)=0, \quad i=1, \ldots, N, \\
& j^{i}\left(x_{i}\left(i^{-}\right), x_{i}\left(i^{+}\right), \lambda_{i}\left(i^{+}\right), \lambda_{i}\left(i^{-}\right), \tau_{i}\right)=0, \quad i=1, \ldots, N, \\
& \mathcal{S}\left(x_{N+1}(N+1), \lambda_{N+1}(N+1)\right)=0, \\
& x_{1}(0)-x_{0}=0 .
\end{aligned}
$$

The jump times $\tau_{i}, i=1 \ldots, N$, appear as unknown variables.

To handle the case $\tau_{N}=T$ we introduce the (unknown) variables

$$
\begin{aligned}
& \mathrm{xT}:=x_{N+1}\left(T^{+}\right), \\
& \mathrm{lT}:=\lambda_{N+1}\left(T^{+}\right),
\end{aligned}
$$

together with the additional boundary conditions

$$
\begin{aligned}
& j^{x}\left(\mathrm{xT}, x_{N+1}(N+1), \mathrm{lT}, T\right)=0, \\
& j^{\lambda}\left(x_{N+1}(N+1), 1 \mathrm{~T}, \lambda_{N+1}(N+1), T\right)=0,
\end{aligned}
$$

and replace (30f) by

$$
\mathcal{S}(\mathrm{xT}, \mathrm{lT})=0 .
$$

The case $\tau_{1}=0$ can be treated in an analogous way. We therefore set

$$
\begin{aligned}
& \mathrm{x} 0:=x_{1}\left(0^{+}\right), \\
& 10:=\lambda_{1}\left(0^{+}\right),
\end{aligned}
$$

together with the additional boundary conditions

$$
\begin{aligned}
& j^{x}\left(\mathrm{x} 0, x_{0}, 10,0\right)=0, \\
& j^{\lambda}\left(x_{0}, 10, \lambda_{1}(0), 0\right)=0,
\end{aligned}
$$

and replace $(30 \mathrm{~g})$ by

$$
x_{1}(0)-\mathrm{x} 0=0 .
$$

During the continuation process it may be of interest to determine the exact value of end time $T$ where the solution jumps at the end time and additionally the condition (30e) is satisfied. In general this characterizes the crossing from a jump at the boundary 
to an interior jump. For that case the time horizon $T$ is considered as a free variable and the condition

$$
j^{N+1}\left(x_{N+1}(N+1), \mathrm{xT}, \lambda_{N+1}(N+1), \mathrm{lT}, T\right)=0,
$$

is appended to (31).

Initializing the $B V P$ To find the solution of a specific problem of type (1) we can apply a continuation strategy with respect to the time horizon $T$. Therefore, as a first step we have to determine an initial (optimal) solution.

Due to Assumption 1, the initial condition together with the transversality condition yield the necessary equations for $T=0$. This solution can be used as a starting point for paths, which for a "small" time horizon do not exhibit a jump point.

\section{Endogenous lumpy investments}

When a firm is dealing with market power, the output price decreases with the quantity that is produced. Since it holds in this model that with one unit of capital stock one unit of output is produced, we have that the output price decreases with the amount of capital. So during the time period between two investments the output price increases, since depreciation decreases capital stock. ${ }^{5}$ We consider no scrapping, partial scrapping and total scrapping, i.e. we consider $\gamma=0, \gamma=0.5$ and $\gamma=1$. We provide a numerical analysis starting with the parameter values

$$
b=\frac{1}{n} \log 2=\frac{1}{2} \log 2, \quad \alpha=0, \quad \beta=0.2, \quad C=2 \quad r=0.04, \quad \delta=0.2,
$$

which we adopt as the benchmark throughout this paper. As in Grass et al. (2012), we base our value for $b$ on Moore's law, ${ }^{6}$ where the value for $b$ is such that the efficiency of the technology doubles every $n$ years where we put $n=2$. The results of the first ten investments, are presented in Table 1 for $T=100$. It turns out that the number of investments, $N$, undertaken by the firm is $N=40$ for $\gamma=1, N=49$ for $\gamma=0.5$ and $N=59$ for $\gamma=0$.

Ignoring the first and last investment, we see that the better the technology is, the larger the investment becomes. It seems as if the firm delays the first investment (compared to the others) to start production of a new good. In Fig. 1a this is clearly seen (also see Figs.4a, 6a in "Appendix"). To understand what happens with the first investment we have to distinguish between $\gamma<1$ and $\gamma=1$. When $\gamma<1$ capital growth is increased with each investment without fully scrapping the old capital stock. Because there is only limited scrapping, at an early stage the firm undertakes a relatively high investment to start production. A firm only undertakes this relatively

\footnotetext{
5 Capital decreases due to depreciation via (5) this causes output to decrease and finally (4) implies that output price increases.

6 Moore's law still holds, The Economist, July 14th 2012, Chipping in: A deal to keep Moore's law alive.
} 
Table 1 First ten investments of Impulse Control solutions for $\gamma . T=100$ and parameter values $r=0.04$, $\delta=0.2, b=\frac{1}{2} \log 2, \beta=0.2, \alpha=0 . C=2, K_{0}=0$ and $\theta(0)=1$

\begin{tabular}{llll}
\hline & $\gamma=0$ & $\gamma=0.5$ & $\gamma=1$ \\
\hline$\left(\tau_{i} . I\left(\tau_{i}\right)\right)$ & $4.1651: 1.4877$ & $4.1462: 1.4682$ & $3.8509: 1.3689$ \\
& $7.3464: 1.3571$ & $7.4147: 1.7204$ & $7.1308: 1.9589$ \\
& $10.0022: 1.4032$ & $10.1649: 2.0101$ & $9.9511: 2.4614$ \\
& $12.3693: 1.4610$ & $12.6433: 2.2785$ & $12.5559: 2.9262$ \\
& $14.5474: 1.5188$ & $14.9499: 2.5312$ & $15.0389: 3.3716$ \\
& $16.5895: 1.5751$ & $17.1370: 2.7731$ & $17.4476: 3.8067$ \\
& $18.5276: 1.6299$ & $19.2361: 3.0070$ & $19.8100: 4.2365$ \\
& $20.3835: 1.6837$ & $21.2682: 3.2353$ & $22.1437: 4.6639$ \\
& $22.1724: 1.7365$ & $23.2479: 3.4594$ & $24.4606: 5.0910$ \\
Revenue (discounted) & $23.9056: 1.7887$ & $25.1861: 3.6805$ & $26.7688: 5.5191$ \\
Investment cost (discounted) & 802.4809 & 790.1920 & 771.3955 \\
Total profit (discounted) & 35.3109 & 67.8103 & 97.6050 \\
\hline
\end{tabular}

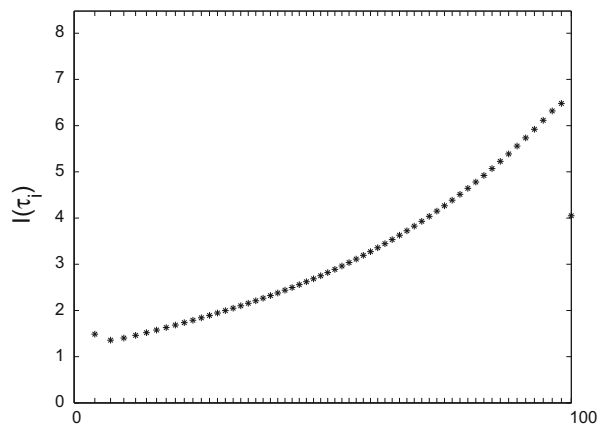

(a)

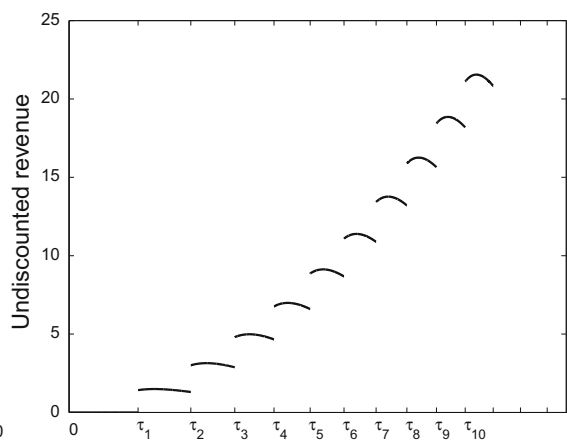

(b)

Fig. 1 For $T=100$ and parameter values $r=0.04, \delta=0.2, \gamma=0, b=\frac{1}{2} \log 2, \beta=0.2, \alpha=0, C=2$, $K_{0}=0$ and $\theta(0)=1$. a Lumpy investments, $I\left(\tau_{i}\right)$. b Undiscounted revenue for the first ten investments

high investment if there is limited scrapping, because the investments help to increase the capital stock in the future.

This behavior is illustrated in Fig. 1a. Drawing a line in the point of Fig. 1a ignoring the first and last investment not only tells us that the first investment is relatively large, but also that the last investment is small. This last investment being small occurs due to the fact that the salvage value of the problem is (too) low, because it does not take into account technological improvement after time $T$.

Table 1 shows that the higher the scrapping percentage the larger the investments become. This makes sense because a firm that wants similar production has to invest extra to replace the scrapped parts. This scrapping increases the investment cost and at the same time, due to the quadratic term in the investment cost function, investing 
Table 2 Technology level and capital for $T=100$ and parameter values $\gamma=0$, $r=0.04, \delta=0, b=\frac{1}{2} \log 2$, $\beta=0.2, \alpha=0, C=2, K_{0}=0$ and $\theta(0)=1$

\begin{tabular}{lrrl}
\hline$\tau_{i}$ & $\theta\left(\tau_{i}^{+}\right)$ & $K\left(\tau_{i}^{+}\right)$ & $\frac{\theta}{K}$ \\
\hline 19.6234 & 7.8009 & 3.8574 & 2.0224 \\
34.5329 & 12.9682 & 6.4650 & 2.0059 \\
50.7184 & 18.5777 & 9.2706 & 2.0039 \\
70.6244 & 25.4766 & 12.7165 & 2.0034 \\
99.7453 & 35.5691 & 17.7443 & 2.0045 \\
\hline
\end{tabular}

such that the same level of capital is reached as in the case of no scrapping, is too expensive. Hence, the optimal level of capital stock in the case of scrapping is lower than under no scrapping, which explains the lower revenue. It turns out that a higher scrapping percentage decreases the number of investments during the planning period.

Another striking effect can be noticed when looking at Fig. 1b. We see that the firm invests in a new product such that marginal revenue is negative. In a "static" model (i.e. a model that does not depend on time) we know that the firms optimize profit and hence invest at the moment that marginal cost is equal to marginal revenue. Since we did not include any operation cost, we know that marginal cost is equal to zero. Hence, when marginal revenue is equal to zero, [i.e. $K(t)=\theta\left(\tau_{i}\right) / 2$ ] investment would be optimal according to this rule. In our dynamic setting it is impossible to stay at the point where marginal revenue is equal to zero, due to depreciation. In Table 2 we show the results for a case where we have no depreciation. We see that indeed the investments are such that the level of capital is set to $K(t)=\theta\left(\tau_{i}\right) / 2$. In the case that we have depreciation, the firm overinvests, i.e., invests such that marginal revenue is negative. Then up until the next investment, marginal revenue increases, becomes zero after some time, and then turns positive.

In Fig. 2 we have plotted the length of the time interval between two investments. We see that in the beginning of the planning period the firm adopts new technologies

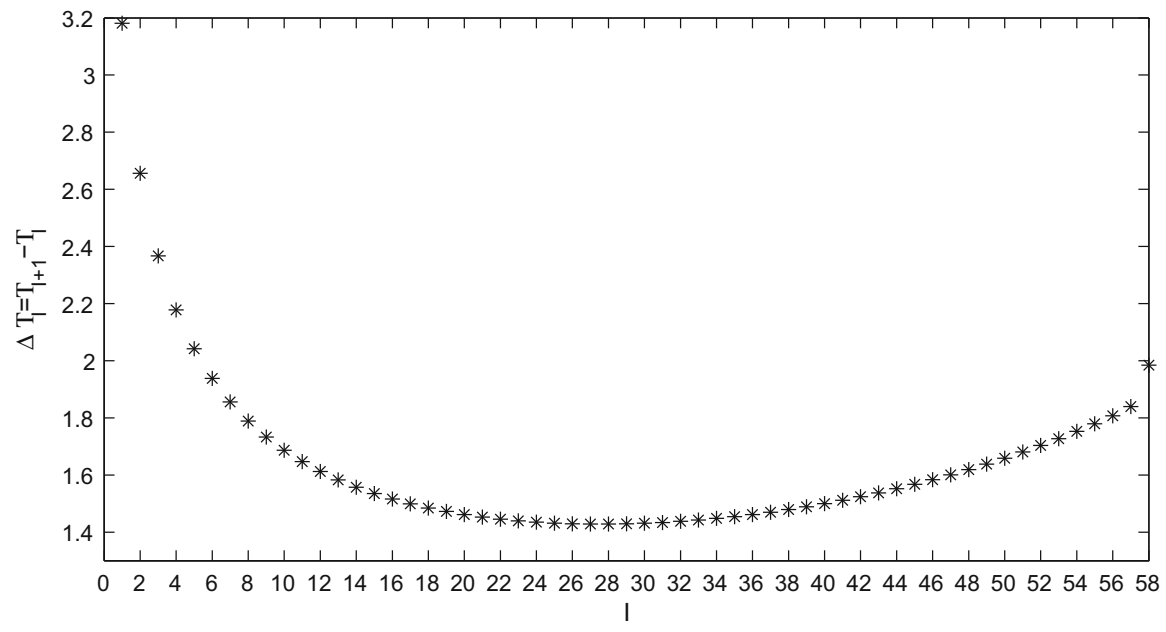

Fig. 2 The length between two investments for $T=100$ and parameter values $r=0.04, \delta=0.2, \gamma=0$, $b=\frac{1}{2} \log 2, \beta=0.2, \alpha=0, C=2, K_{0}=0$ and $\theta(0)=1$ 
faster as time proceeds and after some moment it uses later technologies for a longer time period. This behavior is different from Grass et al. (2012), who finds that the firm adopts new technologies faster as time proceeds for the whole planning period. It is also different from the results found in Saglam (2011), who finds that later technologies are used during a longer time period. Our results are somehow a combination of both. An explanation for this could be that in the beginning of the planning period the firm does not invest much since productivity is low. After some time technological progress is such that each investment is more profitable, which makes that the corresponding capital goods are used for a longer time. For this reason the time between investments increases. Also for higher $T$ a similar effect is found.

\subsection{Sensitivity analysis with respect to the rate of technology change}

Here we study how the rate of technological progress affects the investment behavior of a firm. Remember that we have assumed, using Moore's law, that the efficiency of a technology doubles every $n$ years, setting $n=2$ for our benchmark case. Table 3 shows the first ten investments for different values of the technology rate $b$. In turns out that the number of investments, $N$, undertaken by the firm is $N=45$ for $b=\frac{1}{3} \log 2$, $N=41$ for $b=\frac{1}{4} \log 2, N=38$ for $b=\frac{1}{5} \log 2, N=36$ for $b=\frac{1}{6} \log 2, N=29$ for $b=\frac{1}{10} \log 2$. When $n>5$ an investment takes place at $t=0$. The explanation behind this is that for $n>5$ we have, under Moore's law, that it takes more than five years for the efficiency of a technology to double. Since we have a depreciation rate of $20 \%$, this means that the firm's capital stock is (almost) depreciated before the efficiency of a technology doubles. So the firm has no incentive to wait and invests at $t=0$.

\subsection{Sensitivity analysis with respect to the fixed cost}

One of the main differences between Grass et al. (2012), Boucekkine et al. (2004) and Saglam (2011) is that they do not incorporate any (fixed) cost, whereas this paper assumes that a fixed cost is included for each investment. Here we study how increasing this fixed cost affects the investment behavior of the firm. Table 4 shows the first ten investments for different sizes of fixed cost. In turns out that the number of investments, $N$, undertaken by the firm is $N=44$ for $C=4, N=39$ for $C=8, N=33$ for $C=16$ and $N=27$ for $C=32$. It is easily seen, that if we increase the fixed cost, the first investment is delayed and at the same time the time period between two investments increases. Hence, the number of investments decreases if the fixed cost increases. Comparing the results more carefully, we see that the size of the lumpy investments (i.e. jumps) increases, when $C$ increases.

\section{Lumpy investments under decreasing demand}

In this section we consider the case where the demand for an existing product decreases over time. A main reason could be that the competitors' products become better due to their product innovations. 


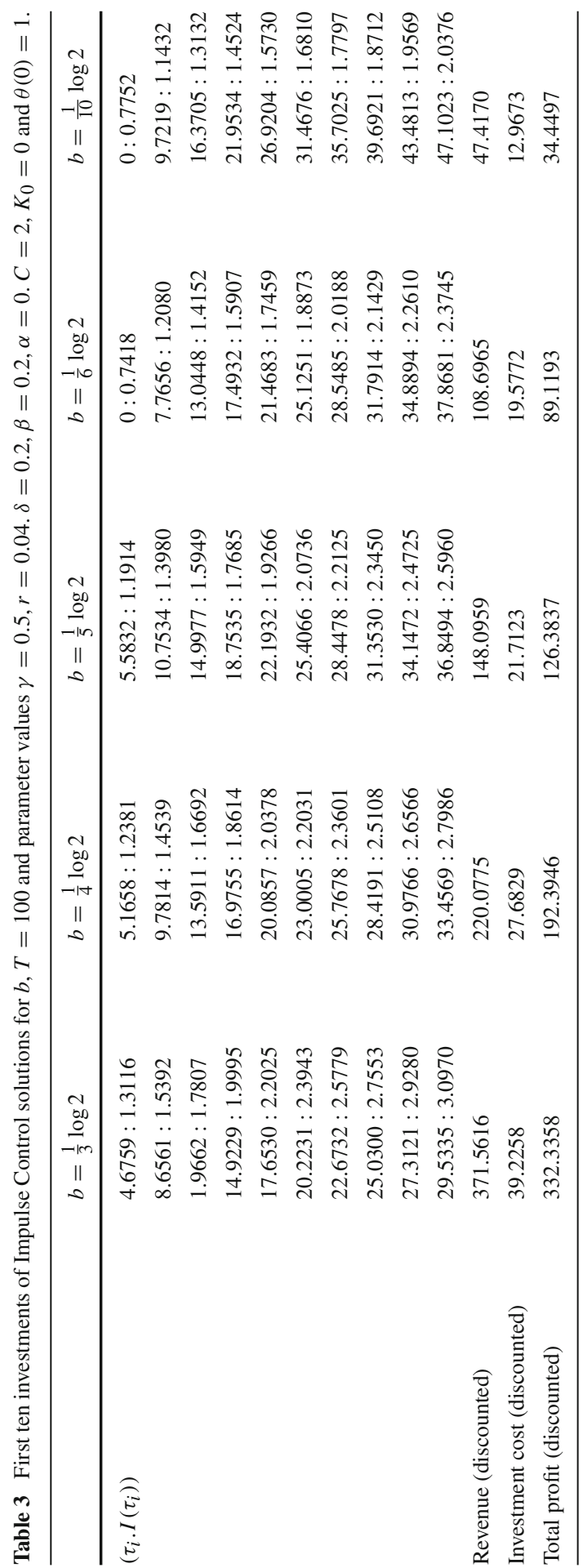


Table 4 Impulse Control solutions for $C, T=100$ and parameter values $\gamma=0.5, r=0.04, \delta=0.2$, $b=\frac{1}{2} \log 2, \beta=0.2, \alpha=0, K_{0}=0$ and $\theta(0)=1$

\begin{tabular}{lllll}
\hline & $C=4$ & $C=8$ & $C=16$ & $C=32$ \\
\hline$\left(\tau_{i} . I\left(\tau_{i}\right)\right)$ & $5.7915: 1.8832$ & $8.0844: 2.4856$ & $11.1517: 3.3199$ & $15.2866: 4.4754$ \\
& $9.6593: 2.2099$ & $12.7147: 2.9206$ & $16.6712: 3.8947$ & $21.8148: 5.2241$ \\
& $12.8816: 2.5607$ & $16.5386: 3.3546$ & $21.1933: 4.4297$ & $27.1293: 5.8789$ \\
& $15.7638: 2.8797$ & $19.9372: 3.7422$ & $25.1901: 4.8993$ & $31.8052: 6.4443$ \\
& $18.4283: 3.1763$ & $23.0621: 4.0984$ & $28.8471: 5.3256$ & $36.0657: 6.9513$ \\
& $20.9394: 3.4571$ & $25.9923: 4.4325$ & $32.2606: 5.7215$ & $40.0266: 7.4169$ \\
& $23.3358: 3.7265$ & $28.7755: 4.7502$ & $35.4889: 6.0947$ & $43.7577: 7.8515$ \\
& $25.6433: 3.9871$ & $31.4435: 5.0556$ & $38.5705: 6.4506$ & $47.3050: 8.2618$ \\
& $27.8799: 4.2412$ & $34.0186: 5.3513$ & $41.5327: 6.7926$ & $50.7012: 8.6525$ \\
Revenue (discounted) & $30.0590: 4.4903$ & $36.5173: 5.6394$ & $44.3957: 7.1237$ & $53.9701: 9.0270$ \\
Investment cost (discounted) & 790.5936 & 769.1875 & 747.0746 & 712.6433 \\
Total profit (discounted) & 701.1899 & 96.8939 & 120.5584 & 150.9987 \\
\hline & & 672.2936 & 626.5162 & 561.6447 \\
\hline
\end{tabular}

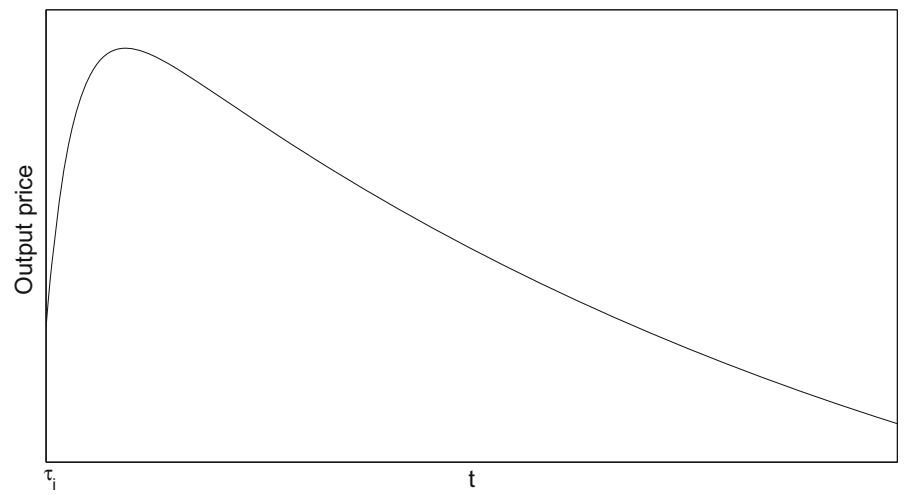

Fig. 3 output price as a function in time for $\delta>\eta$

We incorporate decreasing demand by setting $\dot{\theta}=-\eta \theta(t)$, where $\eta$ is some positive constant. Since it is reasonable to assume $\delta>\eta>0,{ }^{7}$ the output price after investment is first increasing and then decreasing, see Fig. 3. Hence, after a firm invests, capital stock depreciates and the output price first increases, and after some time this output price is decreasing due to this decreasing demand. Then the model becomes

$$
\max _{i, I, \tau_{i}, N}\left\{\int_{0}^{T} e^{-r t}[\theta(t)-K(t)] K(t) d t\right.
$$

\footnotetext{
7 Since we are dealing with product innovation and assume a depreciation rate of $20 \%$ it is unlikely that demand decreases by more than (or equal to) $20 \%$ and hence we do not consider the case that $\eta \geq \delta>0$.
} 


$$
\begin{aligned}
& -\sum_{i=1}^{N} e^{-r \tau_{i}}\left(C+\alpha I\left(\tau_{i}\right)+\beta I\left(\tau_{i}\right)^{2}\right) \\
& \left.+e^{-r T} \frac{\left[\theta\left(T^{+}\right)-K\left(T^{+}\right)\right] K\left(T^{+}\right)}{r+\delta+\eta}\right\},
\end{aligned}
$$

subject to

$$
\begin{aligned}
\dot{K}(t) & =-\delta K(t) \text { for all } t \neq \tau_{1}, \ldots, \tau_{N}, \\
\dot{\theta}(t) & =-\eta \theta(t) \text { for all } t \neq \tau_{1}, \ldots, \tau_{N}, \\
K\left(\tau_{i}^{+}\right)-K\left(\tau_{i}^{-}\right) & =I\left(\tau_{i}\right)-\gamma K\left(\tau_{i}^{-}\right) \text {for all } i=1, \ldots, N \\
\theta\left(\tau_{i}^{+}\right)-\theta\left(\tau_{i}^{-}\right) & =1+b \tau_{i}-\theta\left(\tau_{i}^{-}\right) \text {for all } i=1, \ldots, N \\
K(0) & =0 \\
\theta(0) & =1 .
\end{aligned}
$$

Remember that in Sect. 6 the output price was decreasing in capital stock. Hence, due to depreciation the output price is increasing in the time period between two investments. Since we are considering product innovation, it makes more sense that demand of a given product during the time period decreases. This is because over time new products are invented by other firms, which reduce demand of the current product. This demand decrease has a negative effect on output price and hence the firm has even a greater incentive to invest in a new technology.

In turns out that the number of investments, $N$, undertaken by the firm is $N=40$ for $\eta=0.01, N=35$ for $\eta=0.02$ and $N=31$ for $\eta=0.03$. Looking at the results of Table 5 we can see that a change in the decrease of demand directly affects

Table 5 First ten investments of Impulse Control solutions for $\eta, T=100$ and parameter values $\gamma=0.5$, $r=0.04, \delta=0.2, b=\frac{1}{2} \log 2, \beta=0.2, \alpha=0, C=2, K_{0}=0$ and $\theta(0)=1$

\begin{tabular}{llll}
\hline & $\eta=0.01$ & $\eta=0.02$ & $\eta=0.03$ \\
\hline$\left(\tau_{i} . I\left(\tau_{i}\right)\right)$ & $5.2730: 1.7250$ & $6.3504: 1.9594$ & $7.5126: 2.2042$ \\
& $8.9696: 2.0366$ & $10.4003: 2.3175$ & $11.902: 2.6060$ \\
$12.0850: 2.3821$ & $13.8098: 2.7062$ & $15.5932: 3.0359$ \\
$14.9011: 2.7029$ & $16.8941: 3.0676$ & $18.9345: 3.4366$ \\
& $17.5308: 3.0067$ & $19.7779: 3.4110$ & $22.0629: 3.8188$ \\
& $20.0327: 3.2991$ & $22.5261: 3.7427$ & $25.0493: 4.1897$ \\
& $22.4425: 3.5837$ & $25.1779: 4.0670$ & $27.9368: 4.5542$ \\
& $24.7835: 3.8631$ & $27.7594: 4.3869$ & $30.7539: 4.9156$ \\
Revenue (discounted) & $27.0723: 4.1393$ & $30.2889: 4.7047$ & $33.5212: 5.2765$ \\
Investment cost (discounted) & $29.3212: 4.4136$ & $32.7803: 5.0219$ & $36.2541: 5.6390$ \\
Total profit (discounted) & 762.5966 & 733.2291 & 701.2148 \\
\hline
\end{tabular}


the investment behavior. It is clear to see, that if we increase $\eta$ the first investment is delayed (compared to a smaller $\eta$ ) and at the same time the time period between two investments also increases. Hence, the number of investments decreases if the decay rate of the demand increases. This makes sense, since less demand makes investing less attractive. This results in a lower investment cost for higher $\eta$. Moreover, the larger $\eta$ the lower the output price (compared to a lower $\eta$ ) and hence the lower the revenue.

\section{Conclusions}

This paper employs an Impulse Control modeling approach that is perfectly suitable to take into account the disruptive changes caused by innovations. We describe and implement an algorithm based on current value necessary optimality conditions. The necessary conditions are solved using a multi-point Boundary Value Problem (BVP) combined with some continuation techniques.

Based on our numerical analysis we have derived some guidelines for lumpy investments in new technology:

- A striking result is that the firm does not invest such that marginal profit is zero, but instead marginal profit is negative. Indeed, due to depreciation capital stock decreases in between two investments, implying that marginal profit goes up there due to the decreasing returns to scale assumption. The implication is that during such an interval first marginal profit is negative, but then after a while it turns positive and this stays that way until it is time for the next investment.

- We find that investments are larger and the time between investments is larger when more of the old capital stock needs to be scrapped. If a change in technology permits the firm to keep, update and reuse part of its capital stock, the investments are smaller.

- A nontrivial result is the optimal timing of investments. We see that the firm in the beginning of the planning period adopts new technologies faster as time proceeds, but later on the opposite happens. Moreover, we obtain that the firm's investments increase when the technology produces more profitable products.

- Numerical experiments show that if the time it takes to double the efficiency of a technology is larger than the time it takes for the capital stock to depreciate to half of its original level, the firm undertakes an initial investment.

- Further sensitivity results were provided for a scenario of decreasing demand. We find that when demand decreases over time and when fixed investment cost is higher, then the firm invests less throughout the planning period, the time between two investments increases and the first investment is delayed.

Interesting directions for further work would be to consider running cost in the model or to introduce a learning effect. Another possible extension would be to let the scrapping percentage depend on time.

Open Access This article is distributed under the terms of the Creative Commons Attribution 4.0 International License (http://creativecommons.org/licenses/by/4.0/), which permits unrestricted use, distribution, and reproduction in any medium, provided you give appropriate credit to the original author(s) and the source, provide a link to the Creative Commons license, and indicate if changes were made. 


\section{Appendix: Figures for all cases}

See Figs. 4, 5, 6 and 7.

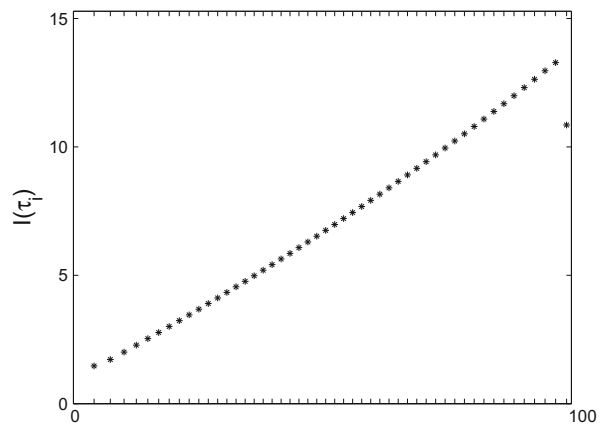

(a)

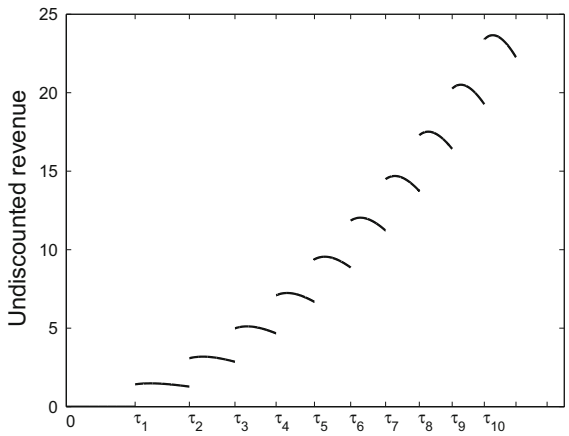

(b)

Fig. 4 For $T=100$ and parameter values $r=0.04, \delta=0.05, \gamma=0.5, b=\frac{1}{2} \log 2, \beta=0.2, \alpha=0$, $C=2, K_{0}=0$ and $\theta(0)=1$. a Lumpy investments, $I\left(\tau_{i}\right)$, b Undiscounted revenue for the first ten investments

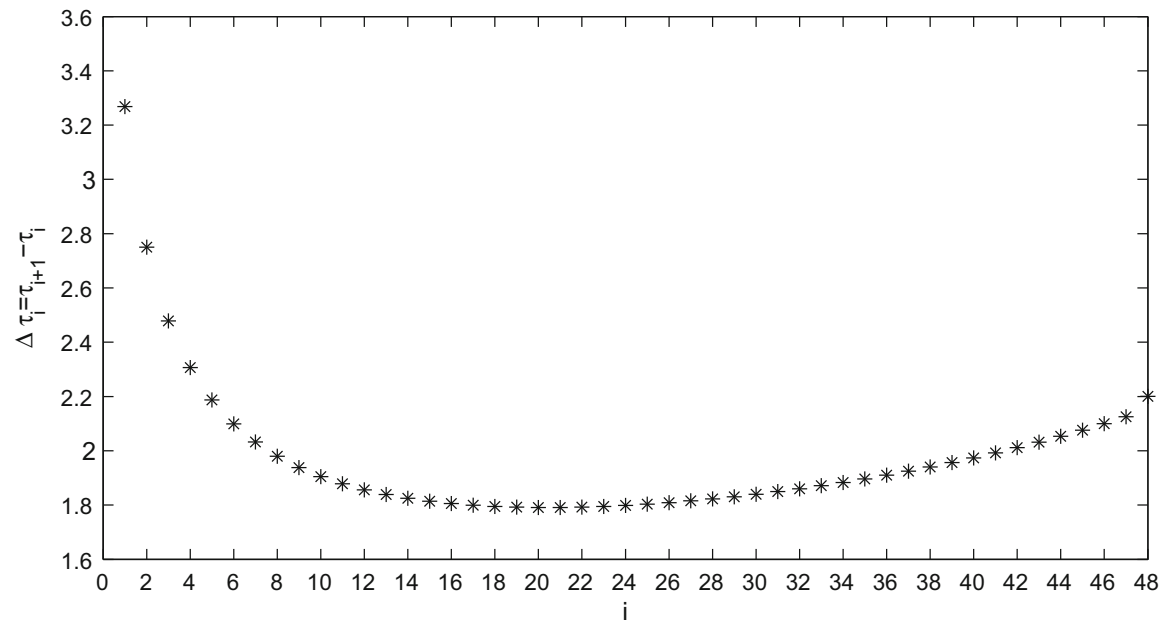

Fig. 5 For $T=100$ and parameter values $r=0.04, \delta=0.2, \gamma=0.5, b=\frac{1}{2} \log 2, \beta=0.2, \alpha=0$, $C=2, K_{0}=0$ and $\theta(0)=1$ 


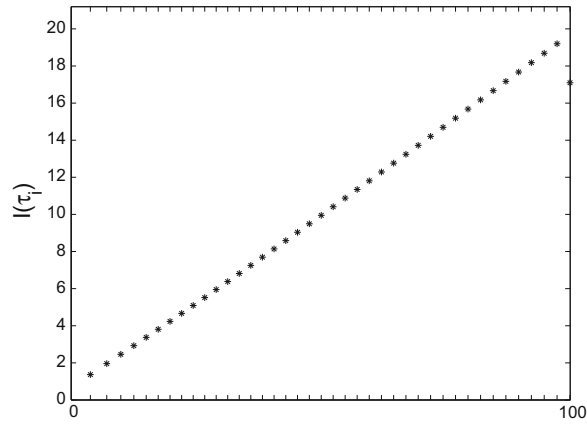

(a)

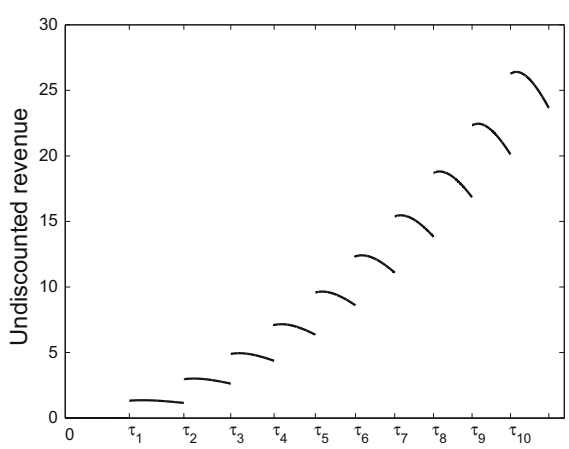

(b)

Fig. 6 For $T=100$ and parameter values $r=0.04, \delta=0.05, \gamma=1, b=\frac{1}{2} \log 2, \beta=0.2, \alpha=0$, $C=2, K_{0}=0$ and $\theta(0)=1$. a Lumpy investments, $I\left(\tau_{i}\right)$. b Undiscounted revenue for the first ten investments

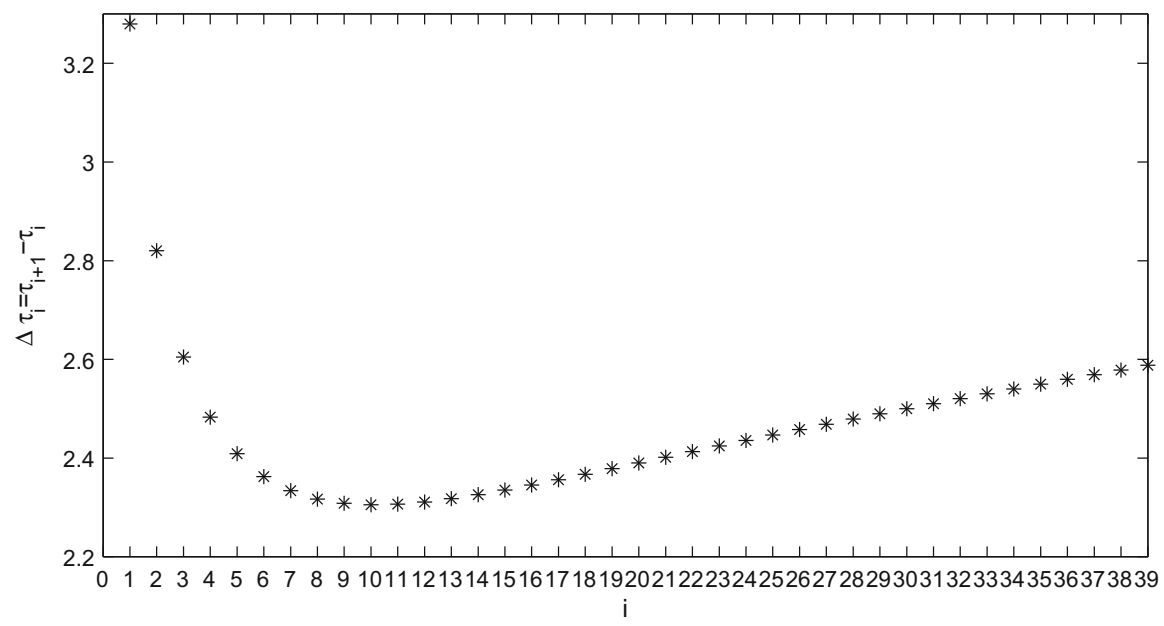

Fig. 7 For $T=100$ and parameter values $r=0.04, \delta=0.2, \gamma=1, b=\frac{1}{2} \log 2, \beta=0.2, \alpha=0, C=2$, $K_{0}=0$ and $\theta(0)=1$

\section{References}

Blaquière A (1977a) Differential games with piece-wise continuous trajectories. In: Hagedorn P, Knobloch HW, Olsder GJ (eds) Differential games and applications. Springer, Berlin, pp 34-69

Blaquière A (1977b) Necessary and sufficient conditions for optimal strategies in impulsive control and application. In: Aoki M, Marzolla A (eds) New trends in dynamic system theory and economics. Academic Press, New York, pp 183-213

Blaquière A (1979) Necessary and sufficient conditions for optimal strategies in impulsive control. In: Lui PT, Roxin EO (eds) Differential games and control theory III, Part A. Marcel Dekker, New York, pp $1-28$

Blaquière A (1985) Impulsive optimal control with finite or infinite time horizon. J Optim Theory Appl 46(4):431-439

Boucekkine R, Saglam C, Vallée T (2004) Technology adoption under embodiment: a two-stage optimal control approach. Macroecon Dyn 8(2):250-271 
Chahim M, Brekelmans RCM, Den Hertog D, Kort PM (2013) An impulse control approach for dike height optimization. Optim Methods Softw 28(3):458-477

Chahim M, Hartl RF, Kort PM (2012) A tutorial on the deterministic impulse control maximum principle: necessary and sufficient optimality conditions. Eur J Oper Res 219(1):18-26

Feichtinger G, Hartl RF, Kort P, Veliov V (2006) Anticipation effects of technological progress on capital accumulation. J Econ Theory 22(5):143-164

Grass D, Chahim M (2012) Numerical algorithm for impulse control models. Working paper, Vienna University of Technology, Vienna

Grass D, Hartl R, Kort P (2012) Capital accumulation and embodied technological progress. J Optim Theory Appl 154(2):588-614

Greenwood J, Hercowitz Z, Krusell P (1997) Long-run implications of investment-specific technological change. Am Econ Rev 87(3):342-362

Kort PM (1989) Optimal dynamic investment policies of a value maximizing firm. Springer, Berlin

Luhmer A (1986) A continuous time, deterministic, nonstationary model of economic ordering. Eur J Oper Res 24(1):123-135

Saglam C (2011) Optimal pattern of technology adoptions under embodiment: a multi stage optimal control approach. Optim Control Appl Methods 32(5):574-586

Seierstad A (1981) Necessary conditions and sufficient conditions for optimal control with jumps in the state variables. Memorandum from Institute of Economics, University of Oslo, Oslo

Seierstad A, Sydsæter K (1987) Optimal control theory with economic applications. Elsevier, Amsterdam

Sethi SP, Thompson GL (2006) Optimal control theory: applications to management science and economics. Springer, Berlin

Yorokoglu M (1998) The information technology productivity paradox. Rev Econ Dyn 1(2):551-592 